\title{
Theoretical and Experimental Demonstration on Grating Lobes of Liquid Crystal Optical Phased Array
}

\author{
Xiangru Wang, ${ }^{1,2}$ Liang Wu, ${ }^{3}$ Man Li, $^{2}$ Shuanghong Wu, ${ }^{1}$ Jiyang Shang, ${ }^{4}$ and Qi Qiu ${ }^{1}$ \\ ${ }^{1}$ School of Optoelectronic Information, University of Electronic Science and Technology of China, Chengdu 610054, China \\ ${ }^{2}$ Science and Technology on Electro-Optical Information Security Control Laboratory, Sanhe 065201, China \\ ${ }^{3}$ School of Physical Electronics, University of Electronic Science and Technology of China, Chengdu 610054, China \\ ${ }^{4}$ Shanghai Aerospace Electronic Technology Institute, Shanghai 201109, China
}

Correspondence should be addressed to Xiangru Wang; xiangruwang@uestc.edu.cn

Received 3 June 2016; Accepted 26 July 2016

Academic Editor: Venkata S. R. Jampani

Copyright (c) 2016 Xiangru Wang et al. This is an open access article distributed under the Creative Commons Attribution License, which permits unrestricted use, distribution, and reproduction in any medium, provided the original work is properly cited.

\begin{abstract}
High deflection efficiency is one of the urgent requirements for practical liquid crystal optical phased array (LC-OPA). In this paper, we demonstrate that high order grating lobes induced from fringe effect are the most important issue to reduce occupation of main lobe. A novel theoretical model is developed to analyze the feature of grating lobes when the device of LC-OPA is working on the scheme of variable period grating (VPG) or variable blazing grating (VBG). Subsequently, our experiments present the relevant results showing a good agreement with the theoretical analysis.
\end{abstract}

\section{Introduction}

Phase controlled steering effects are most often produced by using an array of unit antennas, the amplitude and initial phase of which are individually controllable. Thereby, the technique of phase array (PA) has been suggested to be a wave director from mechanical wave to electromagnetic wave, from microwave to optical domain [1-3]. Meanwhile, phased array has two categories: active PA and passive PA [2]. Active PA has been more widely deployed on the microwave domain because of its easy control and advanced machining accuracy. However, on the optical domain, the wavelength is almost close to 1 micron. To achieve the feature size of submicron, nanoscale processing has already dramatically developed on the optical domain applications, such as adaptive reshaping [4], optical tweezers [5], and optical Yagi-Uda antenna [6]. Even more, an encouraging letter published in Nature in 2013 reported that the first large scale optical phased array (OPA) made by CMOS technology has $64 \times 64$ units [7].

Comparing with active OPA, passive OPA does not have any other heat deposition, such as quantum loss, nonradiation jump, and Joule heat. Since the promotion on the concept of OPA, there have been at least three methods to realize it
$[3,8,9]: \mathrm{LiNbO} 3$, PLZT, and liquid crystal (LC). Therein, the OPA using nematic LC usually called liquid crystal optically phased array (LC-OPA) has a great potential to achieve practical OPA system for steering optical laser beam inertia less, nonmechanical, and low SWaP (Size Weight and Power consumption).

LC-OPA has already demonstrated its properties of light weight and high precision on steering laser beam to generate an optical space-time division multiple access network [10]. After the survival experiment in the equivalent space environment, modest effects were observed, but none were deemed significant enough to impact the performance of the device for beam steering applications on space communication [11]. After the invention of LC-OPA by Dr. McManamon et al. in 1993 [12], he published an all-thing-considered review paper to summarize its nearly 30 years of development [13] including liquid crystal polarization gratings to achieve $99.5 \%$ diffraction efficiency [14], simulation on high efficiency improvement [15], volume holographic to amplify steering angle [16], and the scattering free polymer network liquid crystal invented by Dr. Sun et al. to overcome the transparency reduction on the submillisecond liquid crystal devices $[17,18]$. Meanwhile, the concept of LC-OPA has been 


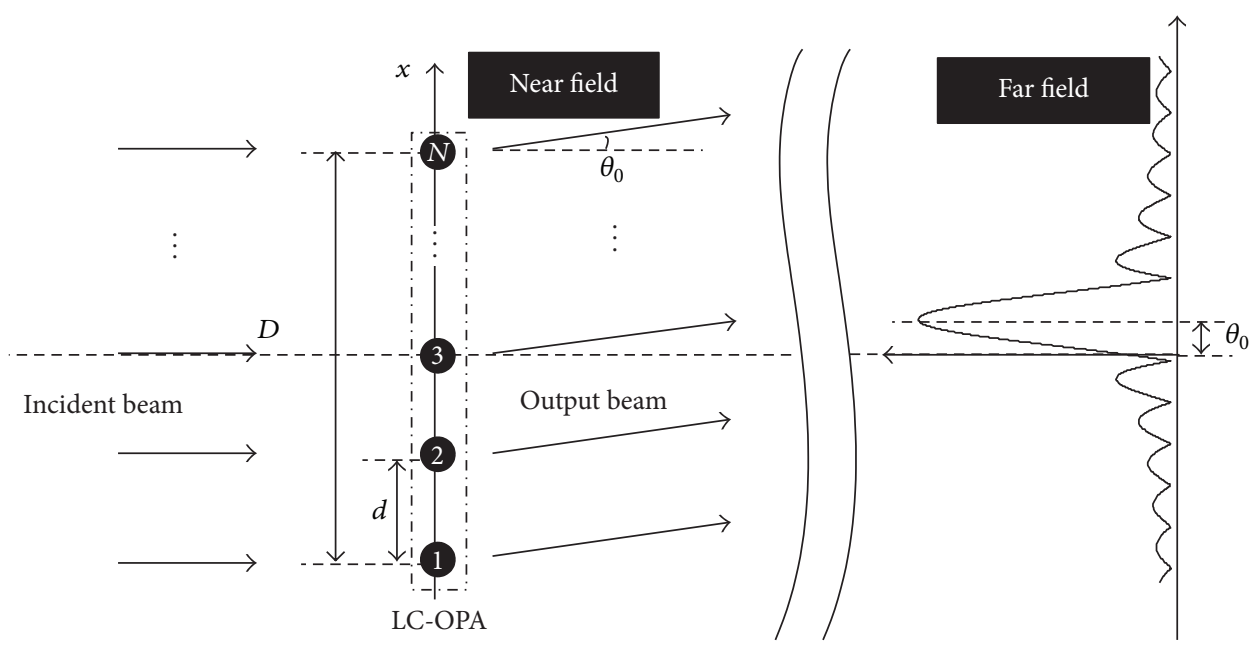

FIGURE 1: The sketch of liquid crystal optical phased array.

suggested into the midinfrared domain [19] and even some alternative methods for steering laser beam such as lenslet array [20] and MEMS-based [21].

Although the performance of LC-OPA has been improved on a variety of features, to date, there has not been a complete analysis on the high order grating lobes. In this paper, it derives a theoretical model on the main lobe and grating lobes and being verified by experimental result.

\section{Theory}

Because of the passive feature of LC-OPA, it has a uniform radiative laser source propagating through the LC film shown in Figure 1. Thereby, the wave front of the polarization dependent near field will be modulated by the liquid crystal film with a gradient refractive index distribution. For the device of LC-OPA, modulation on the amplitude of near field could be neglected because the absorption of working medium is very tiny. After long distance propagation, far field could be generated according to the principle of diffraction so as to a steering peak on the given angular position if a suitable near field phase modulation is given.

Steering can be accomplished by a physical prism. If the refractive index of a prism is changeable by loading different voltages, light could be steered as well at different angles. Meanwhile, we can take advantage of the fact that there is no difference for light waves if they have $0,2 \pi$, $4 \pi$, or $2 k \pi$ phase shift. The phase can be made as a $2 \pi$ subtraction when it exceeds full round $2 \pi$ in the case of large aperture. According to this property of diffraction, it generates the first scheme, shown in Figure 2(a), to form a deflective beam. and the scheme has already been widely used in the current microwave phased array called variable period grating (VPG), where the steering angle $\theta_{s}$ is governed by $\sin \theta_{s}=\Delta \phi_{s} / k_{0} d$, where $\Delta \phi_{s}$ is the phase step between two adjacent electrodes, $k_{0}$ is vacuum wavenumber, and $d$ is width of electrode and gap. Because of the proportional relationship between $\Delta \phi_{s}$ and $\theta_{s}$, it is easy to realize a continuous and full coverage scan on a given domain.

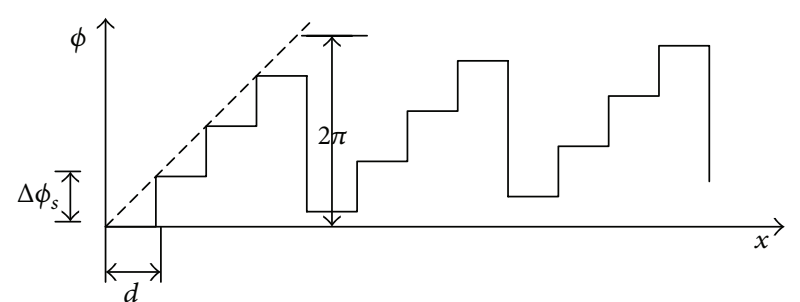

(a)

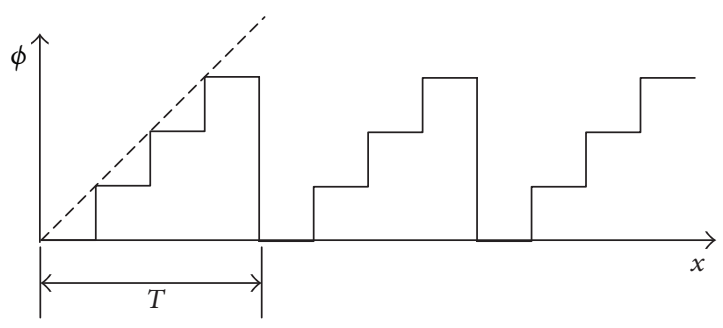

(b)

FIGURE 2: Ideal phase modulation of LC-OPA on the scheme of VPG (a) and VBG (b).

Meanwhile, another scheme to steer wave is blazing grating, shown in Figure 2(b). When an in-plane wave is modulated by periodic medium, tens of periodic spots are generated on the far field that is called grating lobes. Therein, the profile of those grating lobes is determined by the modulation function in one period, and the angular position of the $m$ th order grating lobe $\theta_{m}$ is determined by the grating equation $\sin \theta_{m}=m \lambda_{0} / T$, where $T$ is still the modulation period. When the device is a discrete one, the first order lobe we desire is determined by $\sin \theta_{s}=\lambda_{0} / N d$, where $\lambda_{0}$ is vacuum wavelength and $N$ is the number of electrodes in one period $T$. Comparing with the scheme of VPG, VBG has a constant period $T$. It also always starts at the same initial phase for each period. However, because of the inverse proportional relationship between $\theta_{s}$ and adjustable integer 
$N$, it is impossible to realize a continuous and full coverage scan on one device.

After long distance propagation through the free space, electric field component of the modulated beam is governed by free space Helmholtz equation. If the distance $L$ meets Fraunhofer's approximation condition, that is, $4 L \lambda_{0} \gg D^{2}$, where $D$ is the diameter of transmitting aperture, $E$ field in the far field $E_{\text {far }}$ is completely determined by the near field $E_{\text {near }}$ according to the Fraunhofer's equation

$$
E_{\text {far }}\left(\theta_{x}\right)=A \int_{-\infty}^{+\infty} E_{\text {near }}(x) \exp \left(-j k_{0} \sin \theta_{x} x\right) d x,
$$

where the constant $A$ is determined by the principle of energy conservation and $\theta_{x}$ is the angular spectrum position. Thereby, the far field distribution can be obtained from a given complicated near field by the numerical method of FFT (Fast Fourier Transformation).

$E$ field on the near field is the transmitted beam after the liquid crystal film,

$$
E_{\text {near }}=E_{\mathrm{in}} \cdot T_{a} \cdot T_{p},
$$

where the incident beam $E_{\text {in }}$ is usually configured as a standard TEM00 in-plane wave or Gaussian mode with a half beam waist of $\omega, E_{\text {in }}=\exp \left(-x^{2} / \omega^{2}\right)$. The transmission function includes two parts: amplitude factor $T_{a}$ and phase factor $T_{p}$. The amplitude factor $T_{a}$ is only a gate function owing to the limited transmitting aperture with a full width of $D_{t}, T_{a}=\operatorname{rect}\left(x / D_{t}\right)$. The phase factor $T_{p}$ is determined by the electric controlled liquid crystal film, $T_{p}=\exp [j \phi(x)]$, where $\phi(x)$ is additional phase retardation. According to the characteristic of Fourier transformation, far field distribution $E_{\text {far }}$ can be rewritten as the convolution of two parts that result from gate limited Gaussian beam and phase modulated transmission function, respectively,

$$
E_{\mathrm{far}} \sim \operatorname{FFT}\left\{E_{\mathrm{in}} \cdot T_{a}\right\} \otimes \operatorname{FFT}\left\{T_{p}\right\} .
$$

The shape of main lobe and side lobe is determined by FFT $\left\{E_{\text {in }} \cdot T_{a}\right\}$, having the same result as previous theories. That is, the full width of main lobe is $\Delta \theta_{\text {main }}=2 \lambda_{0} / D_{t}$ for an in-plane wave through an aperture with a width of $D_{t}$. If the beam waist $\omega$ of incident Gaussian beam is much smaller than the aperture, the full width of main lobe is $\Delta \theta_{\text {main }}=4 \lambda_{0} / \pi \omega$.

Because of the property of transmission function $T_{p}$, a pure phase modulation, if it is a periodic function in the ideal model of LC-OPA, its Fourier transformation must be a group of delta functions, the center position of which is determined by $\operatorname{FFT}\left\{T_{p}\right\}$.

The periodic transmission factor $T_{p}$ can be expanded on the Fourier series

$$
T_{p}=\sum_{n=-\infty}^{+\infty} A_{m} \exp \left(j \cdot m \cdot \frac{2 \pi}{T} \cdot x\right),
$$

where the integer $m$ is the order number and $A_{m}$ is determined by the equation

$$
A_{m}=\frac{1}{T} \int_{0}^{T} T_{p}(x) \exp \left(j \cdot m \cdot \frac{2 \pi}{T} \cdot x\right) d x .
$$

Because $T_{p}$ is a phase modulation function on the form of $T_{p}=\exp [j \phi(x)]$, the coefficient $A_{m}$ is rewritten that

$$
A_{m}=\frac{1}{T} \int_{0}^{T} T_{p}(x) \exp \left[-j\left(m \cdot \frac{2 \pi}{T} \cdot x-\phi(x)\right)\right] d x .
$$

To evaluate the property clearly, a group of parameters are assumed for simulation: electrode width $a=4 \mu \mathrm{m}$, electrode gap $b=1 \mu \mathrm{m}$, electrode length $l=10 \mathrm{~mm}$, and electrode number $M=2000$, that is, electrode period $d=a+b=5 \mu \mathrm{m}$, and transmitting aperture width $D_{t}=M d=10 \mathrm{~mm}$.

In the case of VPG, phase modulation $\phi$ is linear with position $x$ that can be written $\phi=k x$; then, after a distance of $2 \pi / k$, the phase increase $2 \pi$, thereby, $\exp [j \cdot k(x+2 \pi / k)]=$ $\exp (j \cdot k x)$, so that the factor of phase modulation $T_{p}$ is periodic and its period $T$ is $2 \pi / k$, where $k$ is variable. Substituting $\phi(x)$ into (6), the amplitude of each order is coefficient $A_{m}$ and it is obtained that $A_{1}=1$ and $A_{m}=0$ for other orders. So the peak angular position $\theta_{s}$ on the far field can be obtained by $\sin \theta_{s}=k / k_{0}$.

In the case of VBG, a periodic transmission function is that the phase shift is periodic; that is, $\phi(x+T)=\phi(x)$, where $T$ is variable. Owing to the periodic property on phase $\phi$ with a period of $T_{p}$ it is not because of wave property of incident beam, the amplitude of each order is coefficient $A_{m}$ obtained from (6), and the peak angular position $\theta_{s}$ on the far field can be obtained by $\sin \theta_{s}=\lambda_{0} / T$.

Therefore, no matter VPG or VBG, these two schemes can be unified because they have the same reason to steer the incident beam to the given angle. The phase factor of their transmission function has a common property that it is periodic with a period of $T=\lambda_{0} / \sin \theta_{s}$. The difference is that on the scheme of VBG, the period $T$ is a user defined variable. On the scheme of VPG, we configure the slope of phase modulation $k$, and the corresponding period $T=2 \pi / k$.

In the practical LC-OPA, fringe effect has already been evaluated in many papers. Owing to the no source boundary condition on the gap between electrodes, there is not only a flyback area for each $2 \pi$ reset, but also phase dropdown on each electrode gap. Therein, the width of flyback is $L_{b}$. Thereby, the phase shift can be written on the form of $\phi(x)=$ $\phi_{\text {ideal }}(x)+\phi_{\text {fringe }}(x)$, where $\phi_{\text {ideal }}(x)$ is ideal phase modulation, the function of which is linear with position like a saw tooth so as to a periodic transmission function $T_{p}$

$$
\begin{aligned}
& \phi_{\text {ideal }}^{\prime}(x) \\
& \quad= \begin{cases}\phi_{\text {init }}+k_{0} \sin \theta_{s}\left(x-x_{1}\right) & x \in D_{0} \\
\phi_{\text {init }}^{\prime}+\left(k_{0} \sin \theta_{s}-\frac{2 \pi}{T}\right)\left(x-x_{1}^{\prime}\right) & x \in D_{f},\end{cases}
\end{aligned}
$$

where the subdomain $D_{0}$ indicates the ordinary domain and $D_{f}$ indicates the flyback domain. The width of flyback $L_{b}$ is determined by the thickness of liquid crystal device. $x_{1}$ and $x_{1}^{\prime}$ are the starting position and ending position of this period, respectively. $\phi_{\text {init }}$ and $\phi_{\text {init }}^{\prime}$ are their phase at $x_{1}$ and $x_{1}^{\prime}$, respectively. On the scheme of VBG, the distance $\left|x_{1}-x_{1}^{\prime}\right|=$ $T, \phi_{\text {init }}=\phi_{\text {init }}^{\prime}=0$. However, on the scheme of VPG, the phase on the finite width electrode is loaded step by step, phase shift 


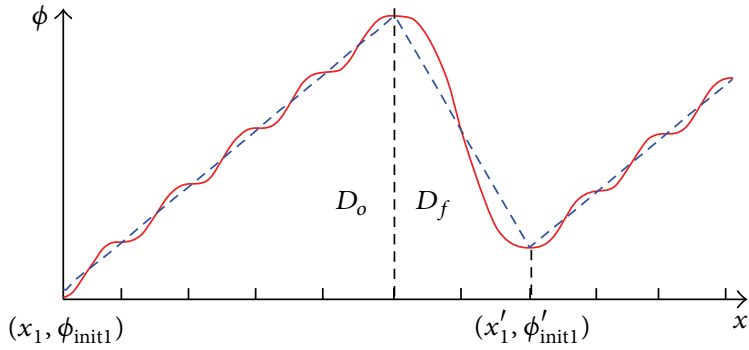

(a)

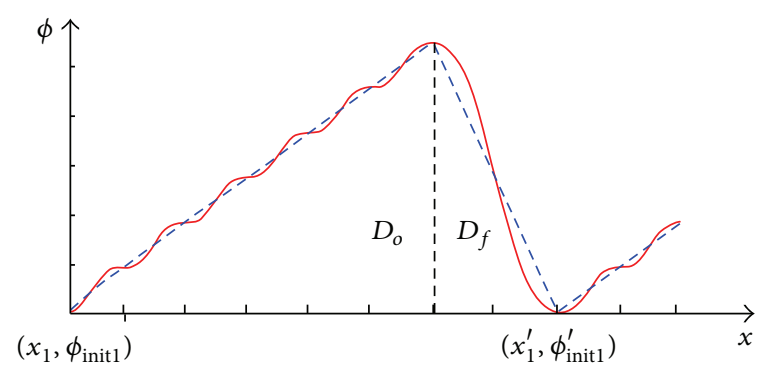

(b)

FIGURE 3: Fringe effect of LC-OPA on the scheme of (a) VPG and (b) VBG.

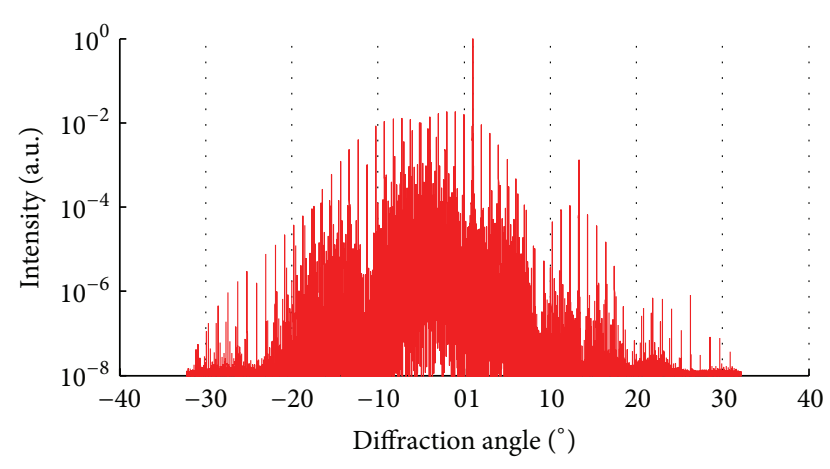

FIGURE 4: Far field of phase modulated by fringe effected LC-OPA.

on the $i$ th electrode $\phi_{i}$ is determined by $\phi_{i}=k(i-1) d$, when it exceeds $2 \pi$, and it would make a reset into $\phi_{\text {init }}=\phi_{i}-2 \pi$ to start another new $2 \pi$ round where the initial phase is $\phi_{\text {init }}$, as shown in Figure 2(a). During the range of one quasi period with $N$ electrodes, the fringe effect induced item $\phi_{\text {fringe }}(x)$ is assumed to be

$$
\phi_{\text {fringe }}(x)= \begin{cases}-p \cdot \sin \left[\frac{2 \pi}{d} \cdot\left(x-x_{1}\right)\right] & x \in D_{0} \\ q \cdot \sin \left[\frac{2 \pi}{d} \cdot\left(x-x_{1}\right)\right] & x \in D_{f},\end{cases}
$$

and because of continuous and desirable feature on phase function, the coefficients $p, q$ should be on the condition of $p:(1-q)=d: L_{b}$. Then, if we assume $p=\Delta \phi_{s} / 2 \pi$, so $q=1-L_{b} / d \cdot \Delta \phi_{s} / 2 \pi$. In the case of VPG, the phase modulation is shown in Figure 3(a); in the case of VBG, it is shown in Figure 3(b). Meanwhile, the values of coefficient and flyback range $L_{b}$ are fully governed by the group of EM equations and liquid crystal molecular director equation.

After the numerical FFT operation on the near field $E_{\text {near }}$, if we suppose steering angle $\theta_{s}$ is 1 degree, the far field is as shown in Figure 4. Comparing with the ideal model with only one lobe on the target angle, there are more grating lobes owing to the additional fringe item. These high order grating lobes are on the position of $\theta=n \theta_{s}$, when $n$ is order number of arbitrary integer.

Therein, the zoom-in figure of Figure 4 is shown in Figure 5; the profile in blue dot line is the FFT of $E_{\text {near }}$ in the range of $[0, T]$, where $T$ is calculated by $2 \pi / k_{0} \sin \theta_{s}$. The intensity of each grating lobe and main lobe at the position

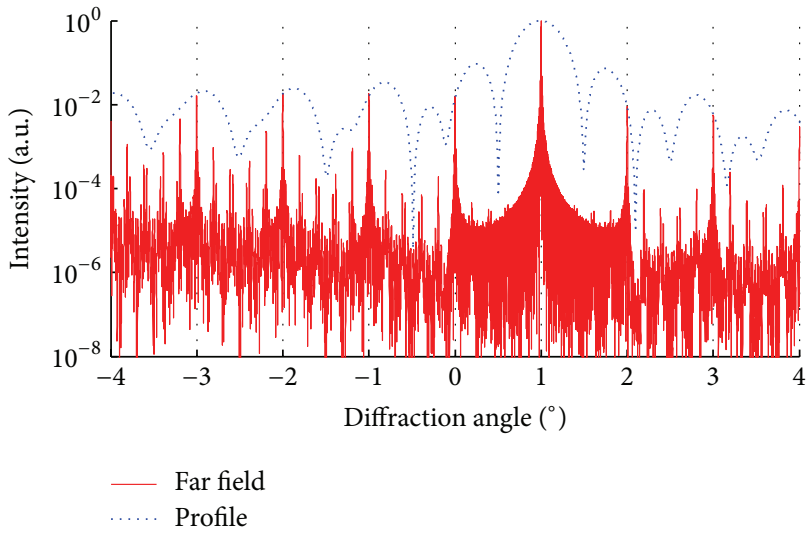

FIGURE 5: Zoom-in figure of normalized intensity of far field of VPG scheme.

of $\theta=n \theta_{s}$ is governed by the blue dot line. Besides the defined higher order grating lobes, there are also some other neglectable peaks like noise. The intensity of most of them is below $30 \mathrm{~dB}$; those are generated by the overlap of multiside lobes.

This property would give us two important issues: first, the phase distribution on the near field can be inversely derived by detecting the intensity and position of each grating lobe; second, by optimizing the phase delay of small account of electrodes in the range of $[0, T]$ can optimize the deflection efficiency. In other words, the phase distribution during one period $[0, T]$ is the major factor to influence the grating distribution or the deflection efficiency.

In the case of VPG, owing to the fringe effect, the transmission factor $T_{p}(x)$ is not perfectly periodic. However, for the most of given value $T_{p 0}, T_{p}$ has a property of repetition on $x$ meeting the equation $\phi(x)=\phi\left(x+X_{q p}\right)$. Meanwhile, the repetition distance $X_{q p}$ is $2 \pi / k_{0} \sin \theta_{s}$; that is, the transmission factor $T_{p}(x)$ is a quasi-periodic function of position, where the period $X_{q p}=2 \pi / k_{0} \sin \theta_{s}$. For the most given value of modulated phase, they would generate a group of grating lobes on the diffraction of $\theta=n \theta_{s}$. And for the phase value which does not have periodic overlap, it would generate the noise lobes. This can be verified in the case of VBG. In contrast, the transmission factor $T_{p}(x)$ is perfectly periodic because of the periodic phase modulation. Then, the far field it generates has a very perfect grating lobes shape 


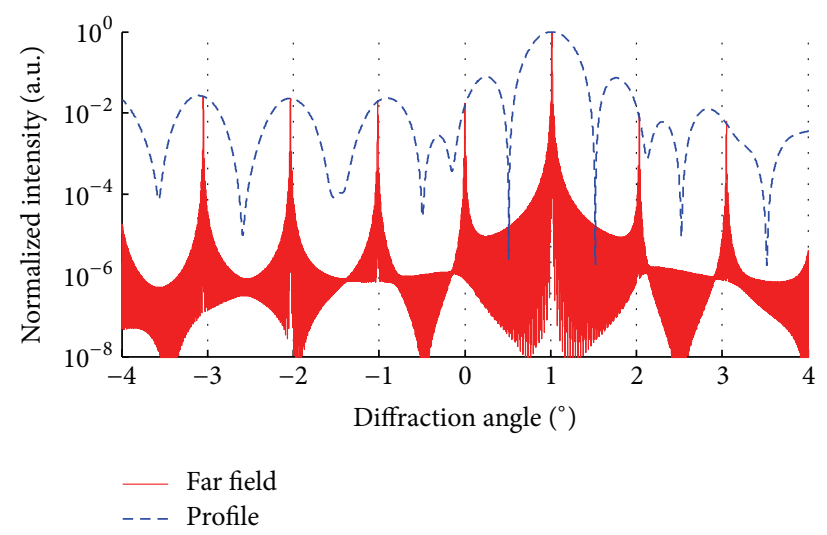

FIGURE 6: Zoom-in figure of normalized intensity of far field of VBG scheme.

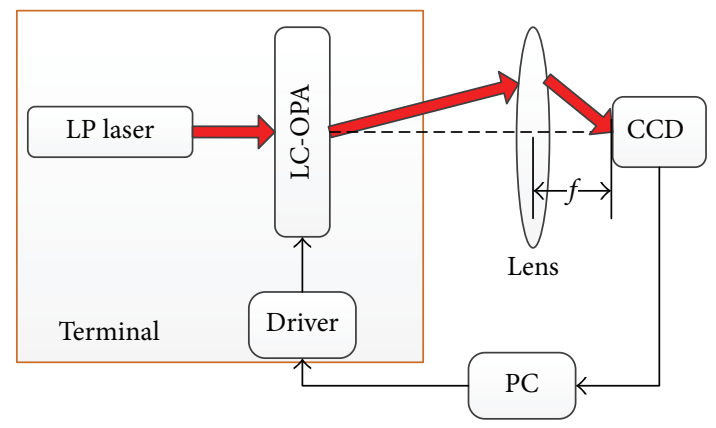

(a)

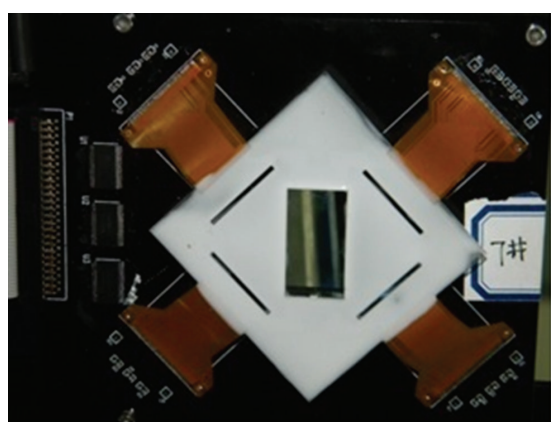

(b)

Figure 7: (a) Experimental setup; (b) LC-OPA phase shifter.

without noise as the VPGs as shown in Figure 6 when the steering angle is 1.02 degrees that is the most close to 1 degree by the grating function $\theta_{s}=\sin ^{-1}\left(\lambda_{0} / N d\right)$.

\section{Experiment}

Figure 7(a) depicts the experimental setup for studying the properties of far field. The incident beam is linear polarized Nd:YAG laser. After phase modulation by the LC-OPA phase shifter device, the output laser beam has a coherent far field observed by a high resolution CCD on the focal plane of Fourier lens. Meanwhile, the data loaded on LC-OPA is generated by PC computer and translated by driver module.

Meanwhile, the device of LC-OPA we used is developed from grating electrodes fabrication to circuit design and liquid crystal filling. The widths of electrode and gap are $4 \mu \mathrm{m}$ and $1 \mu \mathrm{m}$, respectively. The thickness of LC cell is maintained by spacers with a diameter of $10 \mu \mathrm{m}$. The effective optical aperture is $10 \mathrm{~mm} \times 15 \mathrm{~mm}$ as shown in Figure 7(b). Meanwhile, the LC-OPA module is driven by four parallel ordinary liquid crystal display drivers IC controlled by FPGA.

For a given group of steering angle as examples from $-3^{\circ}$ to $3^{\circ}$ with an increasing step of $0.5^{\circ}$, the intensity distributions of far field at those steering angles are captured by CCD and combined together, as shown in Figure 8 from right to left. In order to display the low intensity grating lobe, we adjust the exposure time to the maximum value to detect the grating

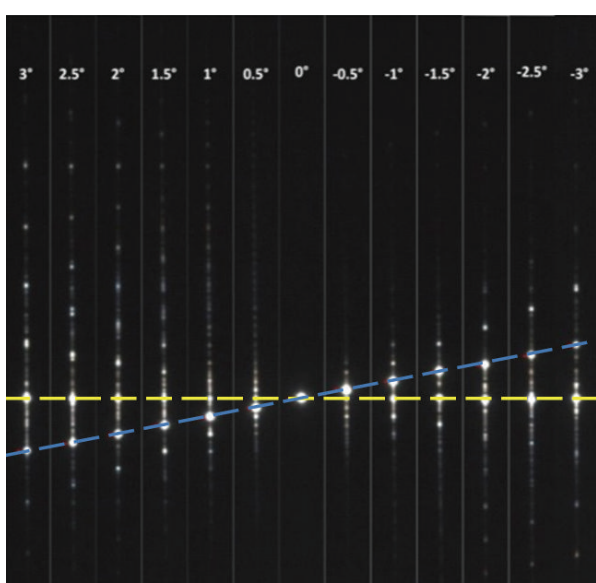

FIGURE 8: Experimental result on the far fields of different steering angles.

lobes and side lobes. The main lobes are lined up by a blue dashed line. They all have good accuracy to form good order on one line. And, the zero-order positions are lined up by the yellow dashed line.

Meanwhile, in Figure 8, grating lobes of higher order are shown with the same distance between each of them. Its distance is the same as the distance between main lobe and 
original position. It has good agreement with the theoretical analysis that $\theta_{n}=n \cdot \theta_{s}$. Besides the grating lobes, Figure 8 also illustrates few relatively dark spots between grating lobes. Although they look very clear in this snapped picture, they do not occupy too much energy when we measured using power meter, because of overexposure.

\section{Conclusion}

Theoretical and experimental evidence is presented showing that grating lobes and side lobes are generated by fringe effect in the far field. On both working schemes of variable period grating (VPG) and variable blazing grating (VBG), the angular positions of grating lobes are determined by $\theta_{n}=$ $n \cdot \theta_{s}$; they have the same physical reason to steer incident beam that their phase factor of transmission function is periodic or quasi-periodic. The normalized intensity of grating lobes is determined by phase modulation in one period or quasi-period. This theoretically and experimentally verified conclusion has been obtained to explain the principle reason on grating lobes.

The principle reason of grating lobes would give us another important issue: optimization on deflection efficiency can be accomplished by optimizing phase delays of small account of electrodes in the range of $[0, T]$, not only VBG scheme but also VPG scheme; higher order and zero order grating lobes can be suppressed as much as possible. Meanwhile, the method of optimization and experimental demonstrations on the steering efficiency improvement is going to be presented in detail in the coming work.

\section{Competing Interests}

The authors declare that they have no competing interests.

\section{Acknowledgments}

This work is sponsored by NSFC Contracts 61405029 and 91438108 and funded by Open Foundation of National Defense Key Laboratory and SAST 2015087.

\section{References}

[1] R. Underbrink J, "Aeroacoustic phased array testing in low speed wind tunnels," in Aeroacoustic Measurements, Experimental Fluid Mechanics, pp. 98-217, Springer, Berlin, Germany, 2002.

[2] R. J. Mailloux, Phased Array Antenna Handbook, Artech House, Boston, Mass, USA, 2005.

[3] P. F. Mcmanamon, T. A. Dorschner, D. L. Corkum et al., "Optical phased array technology," Proceedings of the IEEE, vol. 84, no. 2, pp. 268-298, 1996.

[4] M. Aeschlimann, M. Bauer, D. Bayer et al., "Adaptive subwavelength control of nano-optical fields," Nature, vol. 446, no. 7133, pp. 301-304, 2007.

[5] M. L. Juan, M. Righini, and R. Quidant, "Plasmon nano-optical tweezers," Nature Photonics, vol. 5, no. 6, pp. 349-356, 2011.

[6] T. Kosako, Y. Kadoya, and H. F. Hofmann, "Directional control of light by a nano-optical Yagi-Uda antenna," Nature Photonics, vol. 4, no. 5, pp. 312-315, 2010.
[7] J. Sun, E. Timurdogan, A. Yaacobi, E. S. Hosseini, and M. R. Watts, "Large-scale nanophotonic phased array," Nature, vol. 493, no. 7431, pp. 195-199, 2013.

[8] R. A. Meyer, "Optical beam steering using a multichannel lithium tantalate crystal," Applied Optics, vol. 11, no. 3, pp. 613616, 1972.

[9] J. A. Thomas and Y. Fainman, "Programmable diffractive optical element using a multichannel lanthanum-modified lead zirconate titanate phase modulator," Optics Letters, vol. 20, no. 13, pp. 1510-1512, 1995.

[10] W. J. Miniscalco and S. A. Lane, "Optical space-time division multiple access," Journal of Lightwave Technology, vol. 30, no. 11, pp. 1771-1785, 2012.

[11] S. A. Lane, J. A. Brown, M. E. Tremer et al., "Radiation testing of liquid crystal optical devices for space laser communication," Optical Engineering, vol. 48, no. 11, Article ID 114002, 11 pages, 2009.

[12] P. F. McManamon, E. A. Watson, T. A. Dorschner, and L. J. Barnes, "Nonmechanical beam steering for active and passive sensors," in Infrared Imaging Systems: Design, Analysis, Modeling, and Testing IV, 2, vol. 1969 of Proceedings of SPIE, August 1993.

[13] P. F. McManamon, P. J. Bos, M. J. Escuti et al., "A review of phased array steering for narrow-band electrooptical systems," Proceedings of the IEEE, vol. 97, no. 6, pp. 1078-1096, 2009.

[14] J. Kim, C. Oh, M. J. Escuti, L. Hosting, and S. Serati, "Wideangle nonmechanical beam steering using thin liquid crystal polarization gratings," in Proceedings of the Advanced Wavefront Control: Methods, Devices, and Applications VI, vol. 7093, San Diego, Calif, USA, August 2008.

[15] X. Wang, B. Wang, P. J. Bos, J. E. Anderson, J. J. Pouch, and F. A. Miranda, "Finite-difference time-domain simulation of a liquid-crystal optical phased array," Journal of the Optical Society of America A: Optics and Image Science, and Vision, vol. 22, no. 2, pp. 346-354, 2005.

[16] "Raytheon steered agile beams," STAB, Final Rep. AFRL-SNWP-TR-2004-1078, 2005.

[17] J. Sun, S. Xu, H. Ren, and S.-T. Wu, "Reconfigurable fabrication of scattering-free polymer network liquid crystal prism/ grating/lens," Applied Physics Letters, vol. 102, no. 16, Article ID 161106, 2013.

[18] J. Sun, Y. Chen, and S.-T. Wu, "Submillisecond-response and scattering-free infrared liquid crystal phase modulators," Optics Express, vol. 20, no. 18, pp. 20124-20129, 2012.

[19] Y. Chen, H. Xianyu, J. Sun et al., "Low absorption liquid crystals for mid-wave infrared applications," Optics Express, vol. 19, no. 11, pp. 10843-10848, 2011.

[20] E. A. Watson, W. E. Whitaker, C. D. Brewer, and S. R. Harris, "Implementing optical phased array beam steering with cascaded microlens arrays," in Proceedings of the IEEE Aerospace Conference, vol. 3, pp. 1429-1436, Big Sky, Mont, USA, March 2002.

[21] P. J. Gilgunn and G. K. Fedder, "Flip-chip integrated SOICMOS-MEMS fabrication technology," in Proceedings of the Technical Digest of the Solid-State Sensor, Actuator and Microsystems Workshop, pp. 10-13, Hilton Head Island, SC, USA, June 2008. 

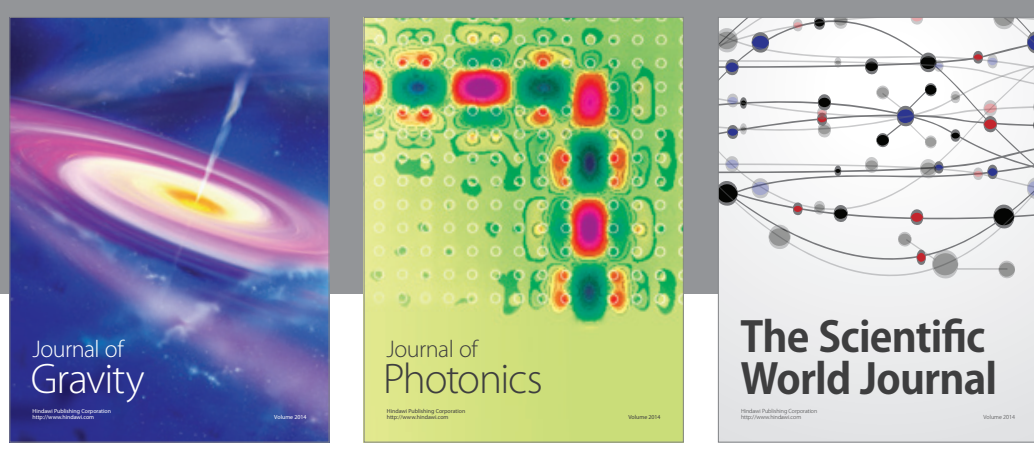

The Scientific World Journal
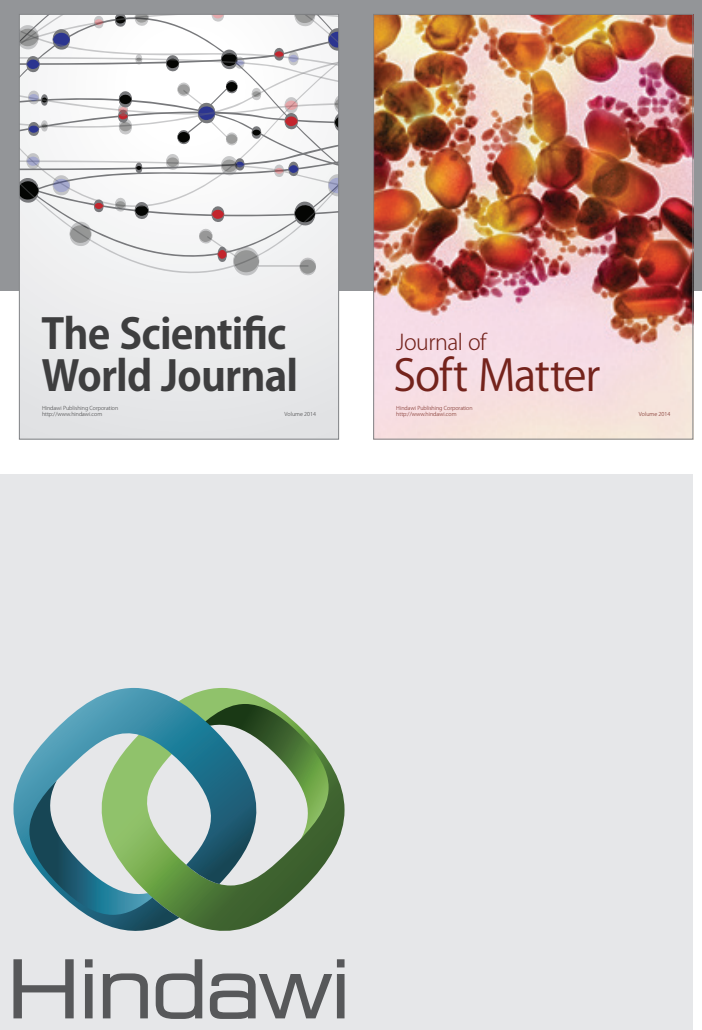

Submit your manuscripts at

http://www.hindawi.com

nternational Journal of

Statistical Mechanics
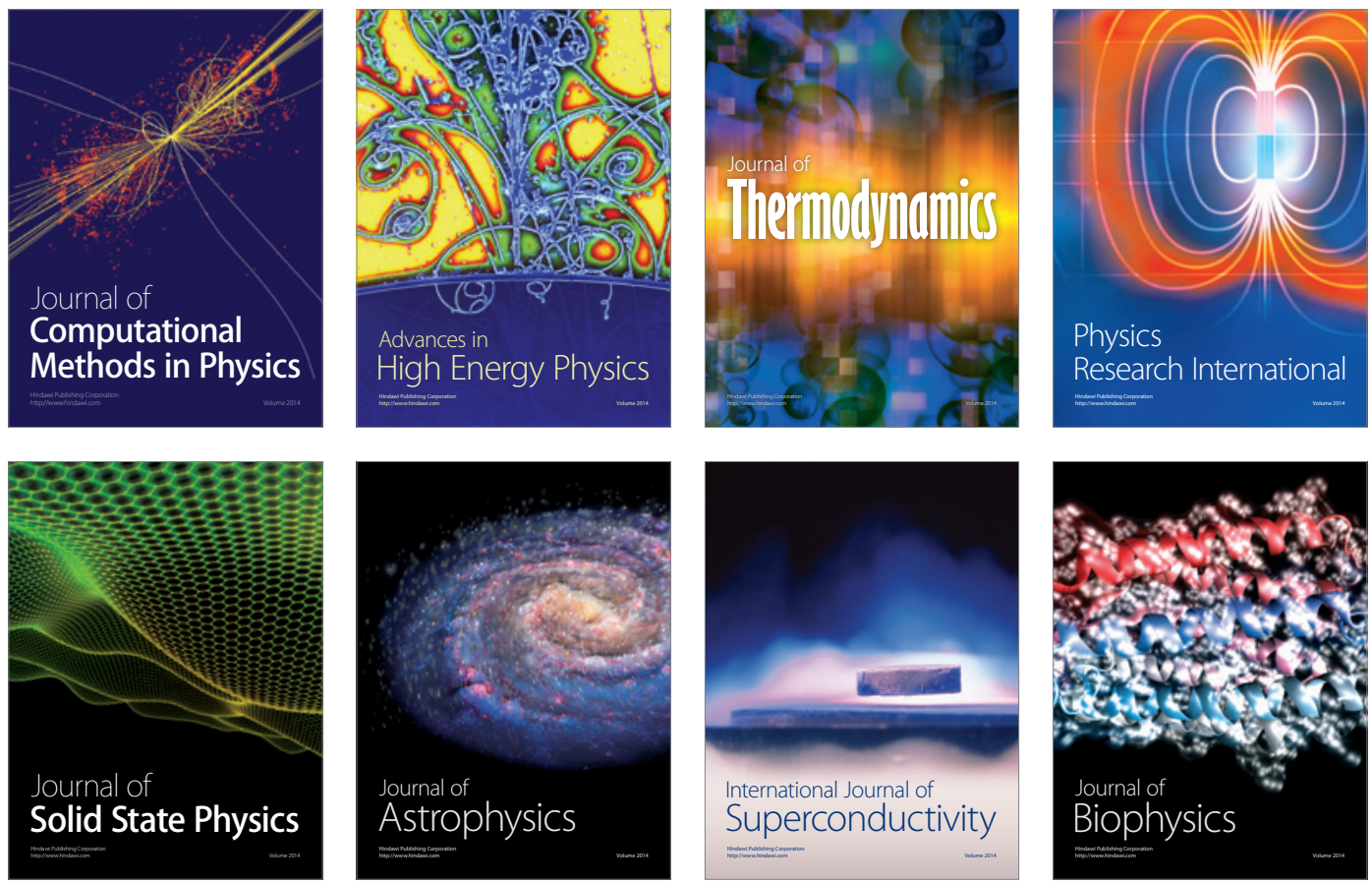
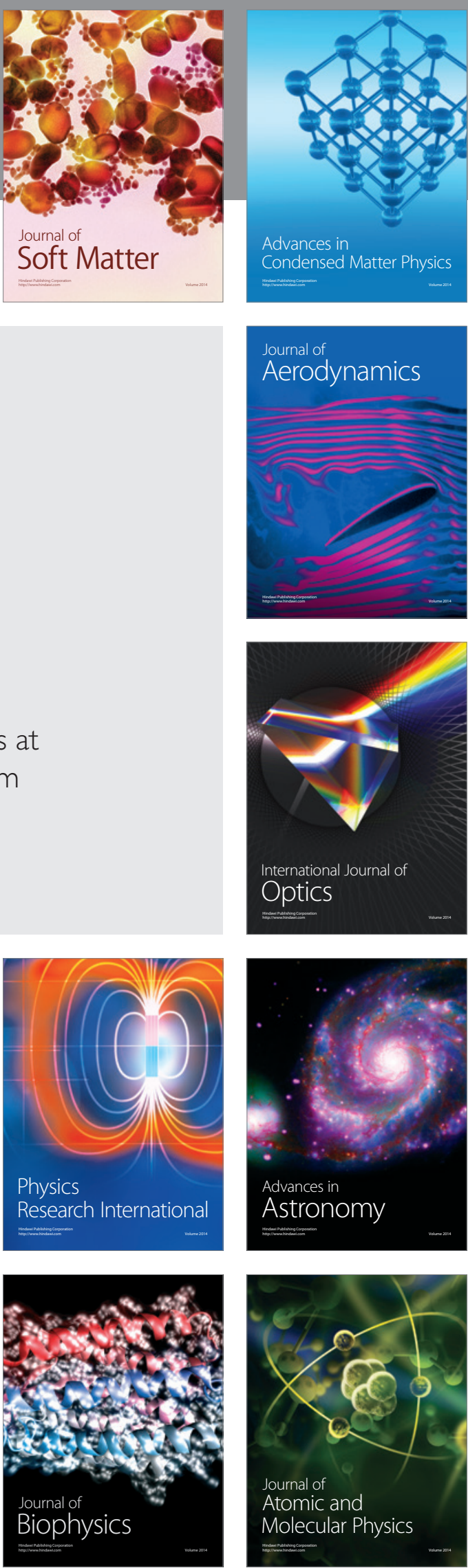